\title{
GO-Kard: An Integrated Documentation System for Cardiology
}

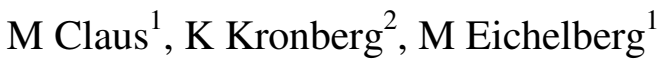 \\ ${ }^{1}$ OFFIS eV, Oldenburg, Germany \\ ${ }^{2}$ Klinikum Oldenburg, Department of Cardiology, Oldenburg, Germany
}

\begin{abstract}
The development of information systems for clinical departments takes a long time, during which the changing requirements from the medical staff lead to a natural growth of the system. Over time, new technologies come and go, leaving their traces in the system architecture. At some point, the system architect needs to think over the whole concept, design a completely new architecture with the most modern technology and re-implement the system as needed. Looking back at more than 15 years of experience in developing software for a clinical cardiology department (exemplarity GO-Kard), this article discusses the rationale of a radical change in the development of a departmental information system and gives a brief explanation of why the time and effort invested in the a new development framework pays off in the long term.
\end{abstract}

\section{Introduction}

GO-Kard, the Graphical Oldenburg Cardiology Information System is an information system for interventional cardiology. It has been developed in cooperation of the Department of Cardiology of the "Klinikum Oldenburg" and the OFFIS research institute over a period of more than 15 years. During this time the system grew in a rather natural way as many improvements were added. Over the years a number of different technologies were used for system development, some of which are no longer supported or for some of which the original producer doesn't even exist anymore. Today the whole system has reached a point where decisions have to be made concerning the future strategy and technological basis. This contribution gives a brief overview of the evolution of the system and discusses reasons for strategic decisions concerning the technologies used.

\section{Historical evolution}

In the year 1989 the Department of invasive cardiology was founded in the "Klinikum Oldenburg". The new head physician had the vision of a computer based documentation system for the catheterization laboratory containing records of all examinations ever made in the cardiological department of the "Klinikum Oldenburg". At that time the main reason was to compute statistics for medical research. Optimizing the workflow and supporting the medical staff was not of prime importance. Computers were not capable of coping with large amounts of multi-media data and concepts for medical information systems, such as [1], were few.

Table 1: Technologies used in GO-Kard

\begin{tabular}{lll}
\hline Year & Software & Hardware \\
\hline 1989 & ORACLE 6 & IBM RS/6000, SUN \\
1989 & ORACLE Forms 2.0 & IBM RS/6000, SUN \\
1992 & C & Apple Macintosh \\
1994 & ORACLE 7 & i386 (Pentium) \\
1995 & ORACLE Forms 4.5 & Apple Macintosh \\
1997 & ORACLE Forms 5.0 & i386 (Pentium) \\
1998 & ORACLE Forms 6.0 & i386 (Pentium) \\
1999 & ORACLE 8i & i386 (Pentium) \\
2000 & C/C++ & i386 (Pentium) \\
2002 & ORACLE 9i & i386 (Pentium) \\
2002 & Java & i386 (Pentium) \\
\hline
\end{tabular}

From the technical point of view a documentation system capable of storing over 300 entries per record - an enormous amount at that time - had to be constructed in a short time of less than six months. PCs were still too slow, so Unix-based systems from IBM and SUN were chosen using relational database technology and front-end tools from ORACLE.

In the first version of the system, the physician filled a paper-based record consisting of six pages after each examination. This record was given to a typist who entered the data in the computer. Trained technicians were required to perform statistical evaluations.

With the availability of fast PCs, especially from Apple, the physicians wanted a system they could use in daily routine. In addition, at that time PCs started to handle pictures. On the technological side this meant writing highly optimized programs and squeezing the last bit of performance out of the existing CPUs. In 1992, the first programs for physicians and medical technicians in the operating room were written in $\mathrm{C}$ with some manually optimized routines in assembler code. However, it 
quickly showed that this kind of source code was very hard to maintain and understand for new co-workers.

At that time, Windows based PCs also became cheaper, faster and as easy to use as Apples Macintosh. Rapid Application Development (RAD) tools for information system development became very popular, so new modules of the system were built with a modern version of ORACLE Forms. Unfortunately, Forms was not well suited for certain requirements. In particular this applied to implementing communication protocols like DICOM (Digital Imaging and Communication in Medicine) or HL7 (Health Level Seven) as well as storage, conversion, retrieval and display of medical films. For this reason, components written in $\mathrm{C}++$ were integrated using different technologies like ActiveX, external program calls and inter process communication.

In Table 1, the introduction of various new development and server platforms over the time is summarized. The database was always an ORACLE relational database management system in different versions while the hardware and the technology for developing the client software $(\mathrm{C}, \mathrm{C}++$, Java, Forms) changed over the years. Always trying to use the most suited technology, different approaches were made to solve problems. Over time this lead to software that could easily be used by the medical staff. No typists or operators were needed anymore.

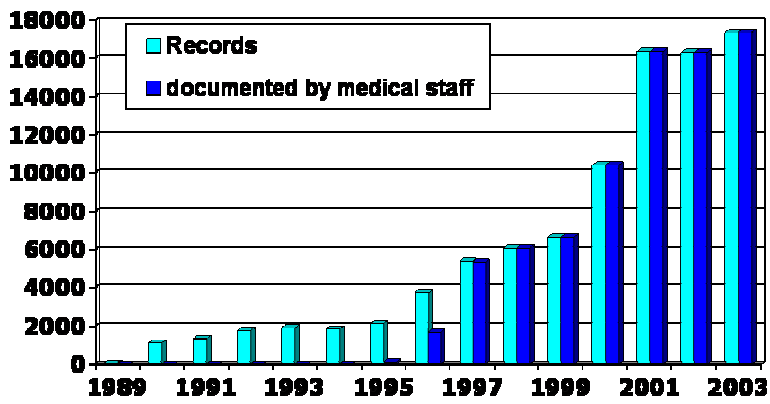

Figure 1: Number of records in the GO-Kard database

Figure 1 shows the number of records (documented cases) in the database in the "Klinikum Oldenburg" for the period 1989 - 2003. Until 1996 the documentation had to be done by a trained typist. After 1996 the software could be used directly by the medical staff, and since 1997 documentation is almost exclusively performed by the medical staff. In 2000 and 2001 new components for ultrasound were added, which explains the sudden rise in the number of cases documented.

\section{Results}

Today, GO-Kard is a complex departmental information system with many features. It supports the documentation of left and right heart catheterization, coronary interventions, peripheral angiography and angioplasty as well as the documentation of various ultrasound and magnetic resonance imaging examinations. It provides users with an automatic report generation, an integrated picture archiving system for storage and retrieval of still images and movies, documentation of used consumables, stock management, and the planning of appointments for patients including written notification generation. The system generates all data required by the German health system and provides data export and statistics facilities for quality control, medical and economical purposes. The system also provides integrated support for documentation of heart surgery. As typical for a departmental information system in cardiology, DICOM interfaces to the imaging modalities are provided, and connection with the central hospital information system (HIS) is made through HL7 interfaces.

However, all of this has not come for free. Over the years the project was faced with a massive integration and communication problem between the parts of the system. One particular problem for the project was the change in ORACLE's philosophy of building database applications. The company made a move away from client-serverarchitectures to completely web-based systems with application servers. In particular this meant that there was no longer support for the classical version of ORACLE Forms available, requiring a re-design of the software on the long term. Finally there was a growing demand for multi-media data. The physicians wanted direct access to all angiography and ultrasound films.

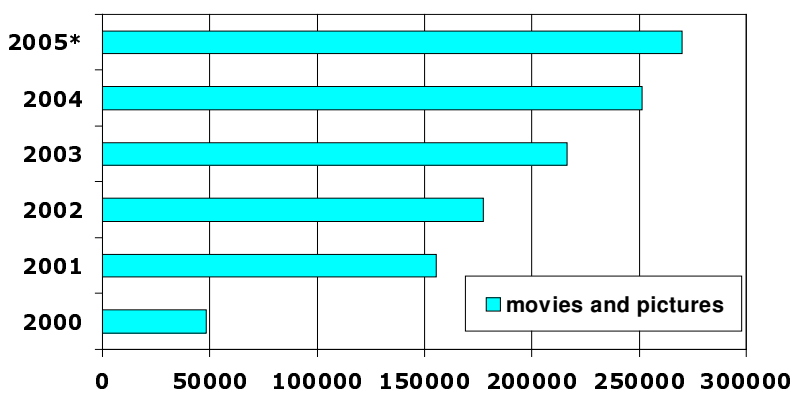

Figure 2: Growing number of objects added annually to the archive

GO-Kard has a fast and sophisticated image archive currently managing more than 2 million films and pictures. Figure 2 shows the increase of the number of movies and still images added annually to the archive (the value for 2005 is estimated). Each record in the archive consists of the multi-media object itself, an overview image and eventually a secondary capture image. Currently more than 2 million movies and pictures are stored in the archive. Most of the movies are not stored in their original DICOM format (which is archived on 
CD-R), but in a compressed format. This means that image quality in the archive is not suitable for the primary diagnosis, which is done directly in the catheterization or echocardiography laboratory anyway. However, in our experience [2,3] the image quality is quite appropriate for review purposes. The compressed storage, however, provides the advantage that the archive despite the large number of films and images currently only occupies about 500 GByte of disk space, and access to individual films is possible within less than one second. On the long term, however, it cannot be ignored that advances in medical imaging techniques lead to ever increasing amounts of data.

From the users standpoint the cardiologists field of work has increased in the last 15 years dramatically. In the cath-lab additional procedures have to be documented like insertion of closure devices in heart defects, implementation of three chamber pacemakers or interventions at great and peripheral vessels. In the noninvasive section new ultrasound modalities like tissue Doppler and contrast echo have been applied. Even new imaging techniques have entered cardiology like magnetic resonance for myocardial and congenital diseases and computer tomography for coronary evaluation. This results in new requirements on the information system.

Adding to these new requirements the problems concerning system integration and projections indicating future performance issues due to the increasing amount of data to be handled raised the question whether or not the current system architecture and development platform was still adequate of purpose. At this stage of the project, our team decided to switch to a modern technology for system development, being convinced that the benefits from a modern platform are high enough to justify the time spent for re-implementing several modules of the existing system. The new technology should meet the following specifications:

- modern, object oriented programming language,

- integrated development environment with editor for graphical user interface and tools for distribution,

- $\quad$ support for the management and presentation of multi-media data,

- $\quad$ proven technology with a good perspective for the long term availability,

- support for the Windows platform necessary, support for Linux/Posix platforms appreciated,

- ease of integration of code written in other programming languages, in particular $\mathrm{C} / \mathrm{C}++$ modules,

- support for a highly modular software architecture.

After some evaluation the team decided on the use of
Micosoft's .NET platform with the C\# programming language and the new Visual Studio 2005 as integrated development environment (IDE) for the new software architecture. This constellation seamed to be most suited for our needs, having advantages over other current development platforms such as Java or $\mathrm{C}++$ in the context of our project. Experience with MONO [4], the Linux port of $\mathrm{C \#}$ and .NET, gave the hope that at some point in time C\#-based applications will run under Linux as well as under Windows with very moderate porting effort. The team also decided to reduce the dependency of the project from third party closed-source modules and runtime environments as far as possible, in order to avoid future experiences similar to the ones following the discontinuation of support for ORACLE Forms. A redesign of the database interface was also planned to enable support for different database management systems including free databases such as PostgreSQL [5]. The main focuses in the development of the new software are a modular design that is giving the user a maximum flexibility for configuring the user interface to his needs, a graphical representation of the anatomy for diagnostic findings and support of all existing and developing cardiological imaging modalities, not only cath-lab and echocardiography.

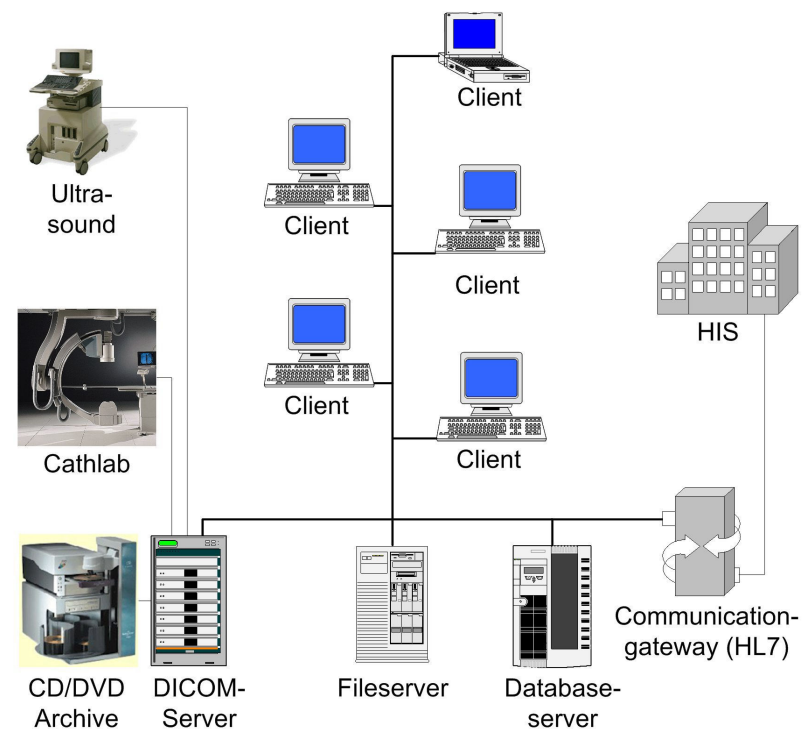

Figure 3: Physical architecture of the GO-Kard system

Figure 3 shows the physical architecture of the GOKard system as implemented today. Each service may be run on a different server system optimized for the specific requirements of that service. In example, the file server is typically optimized for a fast storage subsystem and a fast network connection, while the DICOM server which is responsible for performing the conversion of DICOM objects to MPEG-4 needs much CPU power and system 
memory instead.

On the client side a complete re-design of the software is currently in progress. The client software now consists of small components that can be arranged anywhere in different dialogues, allowing the user to better customize the application to his needs.

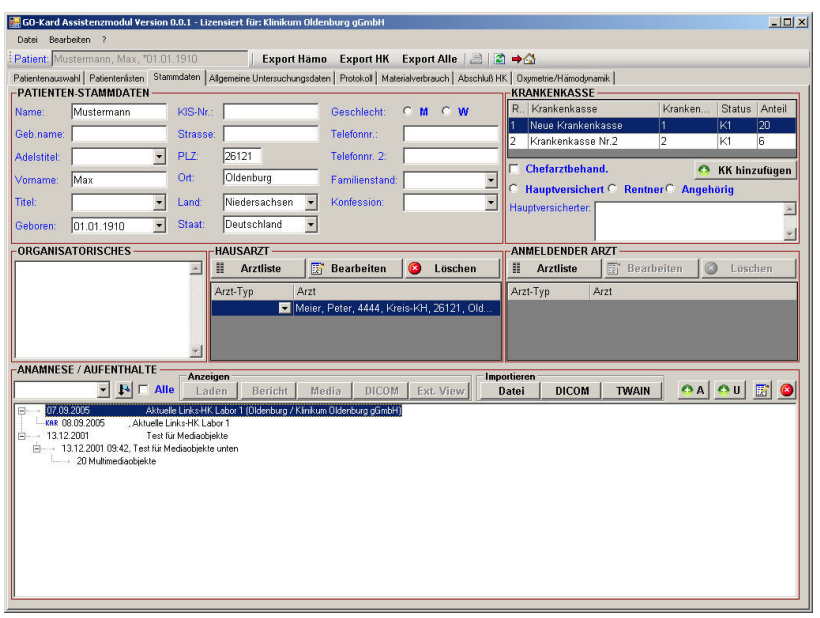

Figure 4: GO-Kard cath-lab module screenshot

Figure 4 shows a screenshot of the software used in the cath-lab. The application in this example consists of eight pages accessible as canvas tabs. The first page holds six components. The configuration for different application profiles is stored in the database and loaded at startup time. Depending on this configuration, the desired number of windows and the window components are instantiated. In a second step the components are assigned to the windows and the first page of the graphical user interface is displayed. The components are independent from each other and communicate through a software-based "communication bus" to which every component is registered during its initialization process. Messages are sent over the communication bus to inform the individual components over user actions and events received from other system components or external systems. A component that "feels responsible" to act on a specific event can respond in an appropriate way. There is a basic GO-Kard software framework that handles most events such as database requests on its own. This allows the developer to focus on the implementation of the desired features.

\section{Discussion and conclusions}

More than 15 years of experience in developing a cardiac information system have led to a stable and practical system that makes documentation easier, faster and more reliable, allowing the doctor and the nurse to concentrate on the medical treatment. The close cooperation between developers and users lead to a software with very high user acceptance.

In the first instance, the re-implementation of a complete software suite means a standstill in progress from the perspective of the user. This holds true in particular in our project since we tried to develop a solid base for future development containing essential but new system functions like an "undo" function, support of tool bars and menu bars in the user interface, a flexible database interface and a highly configurable graphical user interface. This resulted in a rather long planning phase and it took even longer to implement all of the basic features of the software framework. After half a year of intensive work of our team (currently two computer scientist and two programmers working on the system) this paid off in a platform that seems appropriate for our visions. This does not mean that the system is now in a finite and completed state. It rather means that we now have a modern platform, better possibilities for expansion and an easier way of implementing new modules. On the one hand the new framework contains lots of much needed functionalities, so the developer can focus on principal tasks. On the other hand the modular design closes no "doors" for an integration of new techniques.

The experiences with the new architecture are promising. After only six months we were able to release the first prototype, which is currently under test. The team is confident that it was the right decision to change to a completely new technological base. However, before doing so it was essential to look back in history and try to learn out of errors made in the past and things that worked well.

\section{References}

[1] Feigenbaum H: Digital recording, display, and storage of echocardiograms. J Am Soc Echocardiogr; S. 378 - 383; 1988

[2] Kronberg K, Claus M, Riesmeier J, Eichelberg M, Reil $\mathrm{GH}$ : One year experience with a Cardiology PACS using $M P E G-4$ for video compression, in: Proceedings of the 20th EuroPACS annual meeting, Oulu University Press, p. 219 (2002)

[3] Wolfgang Vocke, Anwendung von Videokompressionsalgorithmen zur Datenreduktion koronarangiographischer Untersuchungen, MD Thesis, Medizinische Hochschule Hannover (2002) (in German language)

[4] MONO Homepage: http://www.go-mono.com/

[5] PostgreSQL Homepage: http://www.postgresql.org

Address for correspondence

Marcel Claus

OFFIS e.V., Escherweg 2, D-26121 Oldenburg, Germany

marcel.claus@offis.de 